\title{
Rhabdomyolysis as a Side Effect of the Drug Interaction between Atorvastatin and Sacubitril/ Valsartan
}

\author{
Ka Hong Chan, MD, Payam Pournazari MD, Patrick Champagne MD PhD
}

\author{
About the Authors \\ Ka Hong Chan is with the Department of Medicine, Cumming School of Medicine, University of Calgary. Calgary, AB, Canada. \\ Payam Pournazari and Patrick Champagne are with the Division of Cardiology, Libin Cardiovascular Institute of Alberta and Cumming \\ School of Medicine, University of Calgary. Calgary, AB, Canada. \\ Corresponding author: Patrick.champagne@albertahealthservices.ca \\ Submitted: January 31, 2019. Accepted: March 16, 2019. Published: February 28, 2020. DOI: 10.22374/cjgim.v15i1.343
}

\begin{abstract}
Sacubitril/valsartan is an increasingly used medication in patients with severe left ventricular dysfunction. Here, we present an 83-year-old male with an ejection fraction of $18 \%$ who presented with rhabdomyolysis shortly after initiation of this medication in the setting of being on atorvastatin safely for many years previously. Interestingly, prior pharmacological studies have demonstrated an interaction between sacubitril and atorvastatin via the OATP pathway. In particular, sacubitril has been shown to inhibit OATP1B1 and 1B3, the rate-limiting step for the elimination of atorvastatin, which can result in the drug's accumulation. This phenomenon was determined to be the most likely etiology behind the patient's rhabdomyolysis in this case. Once the medications were discontinued, the rhabdomyolysis resolved. If both a statin and sacubitril/ valsartan need to be co-administered, starting the statin at a low dose with careful monitoring of symptoms, CK, electrolytes, and creatinine during gradual titration should be considered.
\end{abstract}

\begin{abstract}
Resume
Le sacubitril/valsartan est un médicament de plus en plus utilisé chez les patients souffrant de dysfonctionnement grave du ventricule gauche. Nous présentons ici un homme de 83 ans avec une fraction déjection de $18 \%$ qui a présenté une rhabdomyolyse peu après le début de ce médicament dans le cadre d'un traitement à l'atorvastatine sans risque pendant plusieurs années auparavant. Il est intéressant de noter que des études pharmacologiques antérieures ont démontré une interaction entre le sacubitril et l'atorvastatine par la voie de l'OATP. En particulier, il a été démontré que le sacubitril inhibe l'OATP1B1 et 1B3, l'étape limitant la vitesse délimination de l'atorvastatine, ce qui peut entraîner l'accumulation du médicament. Ce phénomène a été déterminé comme étant l'étiologie la plus probable derrière la rhabdomyolyse du patient dans ce cas. Une fois les médicaments arrêtés, la rhabdomyolyse s'est résorbée. Si une statine et le sacubitril/valsartan doivent être co-administrés, il faut envisager de commencer la statine à faible dose en surveillant attentivement les symptômes, la CK, les électrolytes et la créatinine pendant le titrage progressif.
\end{abstract}


Sacubitril/valsartan, an angiotensin receptor-neprilysin inhibitor (ARNI), is a novel drug approved by the United States Food and Drug Administration, Health Canada and the European Commission since 2015 for treatment of congestive heart failure with reduced ejection fraction. Based on the positive results of sacubitril/valsartan over enalapril in the PARADIGM-HF study, both the 2017 American College of Cardiology (ACC) and the Canadian Cardiovascular Society (CCS) guidelines support the use of ARNI in place of a angiotension enzyme converting enzyme inhibitor (ACEi) for patients with advanced heart failure. ${ }^{1-3}$

The reported adverse effects of sacubitril/valsartan during PARADIGM-HF are comparable with ACEi alone, and the current ACC and CCS Guidelines advise to monitor drug tolerability and side effects as with ACEi. ${ }^{1,3}$ Although there is no published data on the proportion of patients on statin therapy in PARADIGM-HF, close to $60 \%$ of the recruited patients had ischemic cardiomyopathy and presumably, would have been on one. Importantly, there are no mentions of rhabdomyolysis, weakness, or falls as potential adverse effects in the trial. ${ }^{2}$ In 2016, the American Heart Association released a statement on drug to drug interactions (DDI) with statins, with particular emphasis on increased risks of muscle toxicity as a consequence. Sacubitril/valsartan was specifically identified as a potential culprit for interacting with statins to cause rhabdomyolysis, even though "no significant adverse events related to atorvastatin were reported" at that time. ${ }^{4}$ This warning can also be found in the pharmaceutical information of sacubitril/valsartan. ${ }^{5}$ Since there has only been one reported case of this drug interaction. ${ }^{6}$ Here, we report another such case as well as review the potential pathophysiology behind this interplay.

\section{Case Report}

An 83-year-old Caucasian man presented with progressive myalgias, dark urine and weakness. The patient's past medical history was significant for non-ischemic dilated cardiomyopathy with an ejection fraction of $18 \%$, dyslipidemia, hypertension, and type 2 diabetes mellitus. His longstanding use of statins had never been complicated by any adverse side effects including myalgias. Medications a month prior to presentation included aspirin $81 \mathrm{mg}$, irbesartan $150 \mathrm{mg}$, spironolactone $12.5 \mathrm{mg}$, atorvastatin $40 \mathrm{mg}$ daily, as well as twice-daily of metformin 1,000 mg, gliclazide $80 \mathrm{mg}$, and metoprolol $25 \mathrm{mg}$. Given the results of the PARADIGM-HF trial and his severe left ventricular (LV) dysfunction, irbesartan was discontinued and sacubitril/valsartan $(24 \mathrm{mg} / 26 \mathrm{mg})$ initiated.

Over approximately three weeks that followed, the patient developed gradually worsening weakness. Following a fall, he presented to the hospital. A review of his recent history was not suggestive of an infectious or inflammatory process, nor was there a history of seizure, heavy exertion, or trauma. Aside from some mild weakness graded at $4 / 5$ in the quadriceps, his neurological examination, including the remainder of his strength testing and reflexes, was otherwise unremarkable. Cardiovascular, respiratory and abdominal examinations were also normal with no right upper quadrant tenderness. He had no signs of compartment syndrome or cutaneous rash. Laboratory investigations demonstrated elevated levels of CK at 16842 $\mathrm{U} / \mathrm{L}$ (Figure 1), lactate dehydrogenase at $586 \mathrm{U} / \mathrm{L}$ and serum creatinine at $162 \mu \mathrm{mol} / \mathrm{L}$. Serum electrolytes (potassium level of $4.3 \mathrm{mmol} / \mathrm{L}$ ) and thyroid-stimulating hormone levels were all within the normal ranges. Moreover, his liver chemistry panel was elevated with a total and direct bilirubin level of 42 and 17 $\mu \mathrm{mol} / \mathrm{L}$, as well as elevated alanine aminotransferase, gammaglutamyl transferase, and alkaline phosphatase levels of 116, 219 and $84 \mathrm{U} / \mathrm{L}$ respectively. Ultrasound of his abdomen was negative for cholelithiasis. Importantly, urinalysis revealed the presence of hematuria on dipstick with no corresponding red blood cells on microscopy, consistent with a diagnosis of rhabdomyolysis.

Given a potential DDI between sacubitril and atorvastatin leading to increased serum concentrations of atorvastatin and hence rhabdomyolysis, both drugs were discontinued. ${ }^{4,6-8}$ As he also had significant left ventricular dysfunction, gentle hydration with occasional diuresis was utilized as his management strategy. With this, his CK gradually returned to normal within a week with coinciding improvements in his mobility (Figure 1). Furthermore, both his acute kidney and liver injuries resolved. He was discharged on irbesartan, with a plan for ongoing medication optimization as an outpatient.

\section{Discussion}

The proposed mechanism behind the DDI is related to the organic anion-transporting polypeptides (OATP), a family

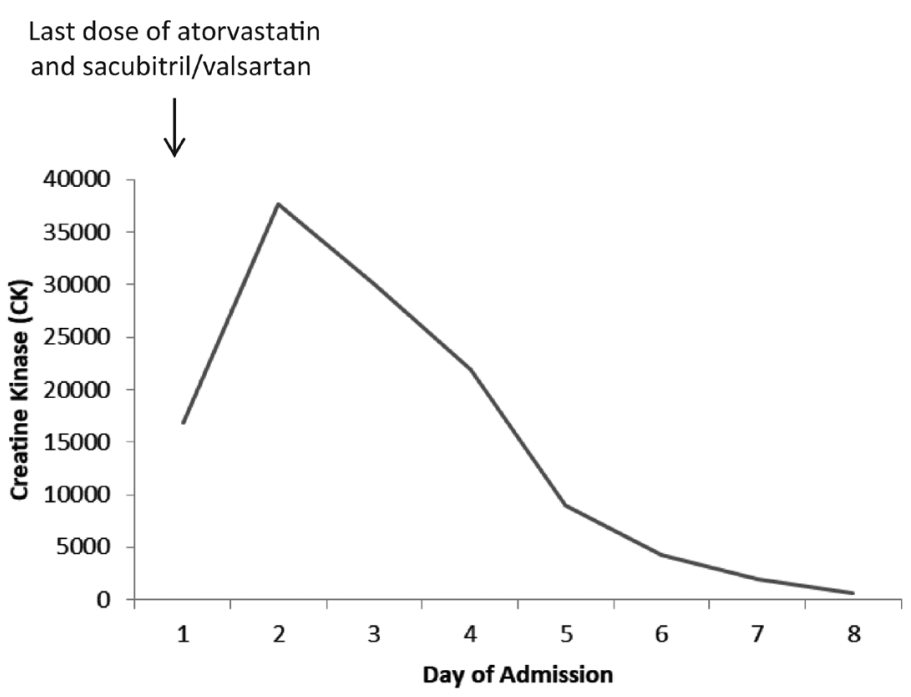

Figure 1. Rhabdomyolysis secondary to interaction between atorvastatin and sacubitril/valsartan with prompt resolution following discontinuation of the medications. 
of transmembrane proteins that facilitate the intracellular transport of substrates into various cells (Figure 2). The OATP family of transporters are found in the basolateral surface of hepatocytes, and are involved in uptake of statins during drug elimination. ${ }^{7,8}$ Importantly, sacubitril has been shown in vitro to inhibit OATP1B1 and 1B3, which can be the rate-limiting step for statin elimination. ${ }^{8}$ This results in up to doubling of the maximum atorvastatin concentration $\left(\mathrm{C}_{\max }\right)$ and an area under the curve (AUC) 1.3 times normal, likely accounting for the clinical findings of rhabdomyolysis as well as liver injury in our patient. ${ }^{8}$ A similar mechanism accounts for the DDI between statins with gemfibrozil, cyclosporine, rifampin and clarithromycin. ${ }^{4}$

This proposed drug interaction had also been studied via a simulation model. ${ }^{8}$ Sacubitril, a prodrug that is the main driver of OATP1B1 inhibition, is rapidly absorbed from the intestines. With a half-life of only 1.4 hours, it is quickly metabolized to its active form sacubitrilat. Therefore, the concentration of sacubitril remaining above the half-maximal inhibitory concentration $\left(\mathrm{IC}_{50}\right)$ of OATP1B1 in the portal vein is approximately 1.8 hours following the dose. ${ }^{8}$ When given concurrently with atorvastatin, which is also rapidly absorbed and have a short time to maximal concentration $\left(\mathrm{T}_{\max }\right)$, the risk for the interaction between the drugs causing rhabdomyolysis can be high. ${ }^{8}$ In particular, our patient was known to take his atorvastatin and sacubitril/valsartan together, which may have predisposed him to an increased risk for this adverse effect.

Further complicating this is the variability of the function of OATP1B1 due to individual haplotypes. A large number of single nucleotide polymorphisms (SNP) in the SLCO1B1 gene encoding for OATP1B1 is common in the general population, with a frequency of $40 \%$ in Europeans and $80 \%$ in Sub-Saharan Africans and East Asians. ${ }^{9}$ A previous study in healthy volunteers

Blood

Hepatocyte

Bile

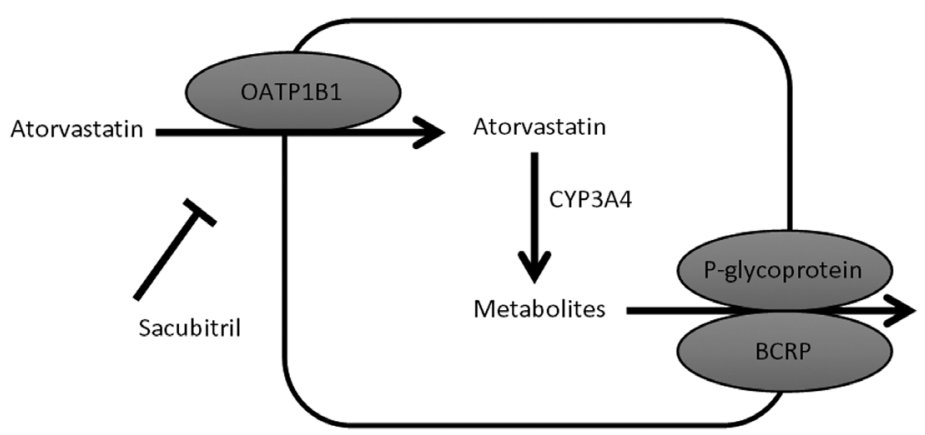

Figure 2. Proposed mechanism between the interaction of atorvastatin and sacubitril/valsartan in hepatocytes during atorvastatin's metabolism and elimination.

$\mathrm{BCRP}=$ breast cancer resistant protein; OATP $=$ organic anion-transporting polypeptides. demonstrated significant increases in the AUC of atorvastatin secondary to $S L C O 1 B 1$ variations in genotypes. ${ }^{10}$ Moreover, ethnic differences in statin concentrations have been attributed to differences in OATP1B1 pharmacokinetics, with higher concentrations found in Asians. ${ }^{11}$ Unfortunately, testing of our patient's SLCO1B1 genotype was not available at the time, but we postulate whether he was also at an increased risk due to this phenomenon. This genetic variability in the function of OATP1B1 may be a potential explanation for the scarcity behind reports of this clinically significant DDI despite prior knowledge of the underlying mechanism. ${ }^{4,6}$ Future studies on the feasibility of screening for this adverse effect, including genetic studies, may be warranted.

Commonly prescribed statins, including rosuvastatin and simvastatin, are also transported via OATP. ${ }^{4,11}$ Interestingly, although simvastatin is the most sensitive statin to the effects of OATP1B1, in vivo studies have shown little notable interaction when co-administrated with sacubitril/valsartan. ${ }^{7,8}$ This may be secondary to simvastatin's longer $\mathrm{T}_{\max }$ at approximately 2 hours relative to atorvastatin, which is hence past the timeframe of the concentration of sacubitril above the half maximal inhibitory concentration of OATP1B1. ${ }^{7,8}$ Clinically, statins with longer $\mathrm{T}_{\max }$, such as simvastatin and rosuvastatin, may therefore be possible alternatives for patients requiring co-administration of the two medications. ${ }^{8}$ Yet, it is also important to acknowledge the large genetic variability in the SLCO1B1 gene that encodes for OATP1B1, and hence, careful monitoring of liver enzymes, electrolytes, creatinine, and CK may be warranted during the initial few weeks of co-administration. ${ }^{9}$ Lowered doses of statins with careful titration should also be considered. ${ }^{5}$

A significant proportion of patients who may derive mortality benefit from sacubitril/valsartan also have a strong indication for the use of a statin. With strong evidence supporting the use of a statin in that population, awareness of a possible DDI between sacubitril and statins, especially atorvastatin, is essential to ensure patient safety. In summary, prescribing physicians and patients ought to be aware of the heightened risk for rhabdomyolysis when these agents are co-prescribed. If co-administration is required, starting the statin at a low dose with careful monitoring of CK, electrolytes, and creatinine during gradual titration should be considered.

\section{Declaration and Conflicts of Interests}

None.

\section{Author Contributions}

Ka Hong Chan: design of case report, drafting and revising the manuscript. Payam Pournazari and Patrick Champagne: design of case report and revising manuscript. 


\section{Study Funding}

No targeted funding reported.

\section{References}

1. Ezekowitz JA, O’Meara E, McDonald MA, et al. 2017 Comprehensive Update of the Canadian Cardiovascular Society Guidelines for the Management of Heart Failure. Can J Cardiol 2017;33(11):1342-3.

2. McMurray JJ, Packer M, Desai AS, et al. Angiotensin-neprilysin inhibition versus enalapril in heart failure. N Engl J Med 2014;371(11):993-1004.

3. Yancy CW, Jessup M, Bozkurt B, et al. 2017 ACC/AHA/HFSA Focused Update of the 2013 ACCF/AHA Guideline for the Management of Heart Failure: A Report of the American College of Cardiology/American Heart Association Task Force on Clinical Practice Guidelines and the Heart Failure Society of America. Circulation 2017;136(6):e137-e61.

4. Wiggins BS, Saseen JJ, Page RL, 2nd, Reed BN, Sneed K, Kostis JB, et al. Recommendations for Management of Clinically Significant DrugDrug Interactions With Statins and Select Agents Used in Patients With Cardiovascular Disease: A Scientific Statement From the American Heart Association. Circulation. 2016;134(21):e468-e95.
5. Entresto (sacubitril/valsartan) product monograph: Novartis Pharmaceuticals Canada; 2017. Accessed on March 31, 2019.

6. Faber ES, Gavini M, Ramirez R, Sadovsky R. Rhabdomyolysis after coadministration of atorvastatin and sacubitril/valsartan (Entresto ${ }^{\mathrm{m}}$ ) in a 63-Year-old woman. Drug Saf Case Rep 2016;3(1):14.

7. Ayalasomayajula S, Han Y, Langenickel T, et al. In vitro and clinical evaluation of OATP-mediated drug interaction potential of sacubitril/ valsartan (LCZ696). J Clin Pharm Ther 2016;41(4):424-31.

8. Lin W, Ji T, Einolf H, et al. Evaluation of drug-drug interaction potential between sacubitril/valsartan (LCZ696) and statins using a physiologically based pharmacokinetic model. J Pharm Sci 2017;106(5):1439-51

9. Kalliokoski A, Niemi M. Impact of OATP transporters on pharmacokinetics. Br J Pharmacol 2009;158(3):693-705.

10. Pasanen MK, Fredrikson H, Neuvonen PJ, Niemi M. Different effects of SLCO1B1 polymorphism on the pharmacokinetics of atorvastatin and rosuvastatin. Clin Pharmacol Ther 2007;82(6):726-33.

11. Kellick K. organic ion transporters and statin drug interactions. Curr Atheroscler Rep 2017;19(12):65. 